\title{
Hierarchical Tone Mapping for High Dynamic Range Image Visualization
}

\author{
Guoping Qiu and Jiang Duan \\ School of Computer Science, The University of Nottingham, United Kingdom
}

\begin{abstract}
In this paper, we present a computationally efficient, practically easy to use tone mapping techniques for the visualization of high dynamic range (HDR) images in low dynamic range (LDR) reproduction devices. The new method, termed hierarchical nonlinear linear (HNL) tone-mapping operator maps the pixels in two hierarchical steps. The first step allocates appropriate numbers of LDR display levels to different HDR intensity intervals according to the pixel densities of the intervals. The second step linearly maps the HDR intensity intervals to theirs allocated LDR display levels. In the developed HNL scheme, the assignment of LDR display levels to HDR intensity intervals is controlled by a very simple and flexible formula with a single adjustable parameter. We also show that our new operators can be used for the effective enhancement of ordinary images.
\end{abstract}

Keywords: High dynamic Range Image, Image Visualization, Tone Mapping, Image Rendering

\section{INTRODUCTION}

Today's digital cameras are very advanced. However, one of the areas where current digital photography technology lacks is dynamic range. The real world scenes we experience in our daily life often have a very wide range of intensity values. Human visual system is capable of perceiving scenes with brightness ranges over five orders of magnitude and can gradually adapt to scenes with dynamic ranges over nine orders of magnitude. The ultimate goals of digital imaging would be to develop systems that match or even exceed the capabilities of human visual system. It is conceivable that future digital cameras would be able to capture high dynamic range (HDR) photos by the click of a button, however, current technology often only enables part of the real world high dynamic scenes visible in any one single shot.

In fact, as it has turned out, recent technologies have made it relatively easy to create numerical radiance maps that capture the full dynamic range of real world scenes [1]. Using the method of [1], a HDR radiance map of a scene can be generated by using a sequence of low dynamic range (LDR) images of the same scene taken under different exposure intervals. Apart from the method of [1], other techniques for capturing HDR images have also been developed by various researchers e.g., [2] and [3]. More recently, technology has also been developed to capture high dynamic range videos [4].

The HDR radiance map records the full brightness dynamic range of the scene in numerical format. However, most visualization/reproduction devices, such as CRT monitors or printers, can only reproduce images with a dynamic range spanning no more than a few orders of magnitude, which is significantly lower than the dynamic range of the radiance map data. In order to reproduce HDR maps in LDR devices, mapping or tone reproduction techniques are used to map HDR values to LDR values. Tone reproduction (mapping) is a crucial step in high dynamic range digital imaging workflow, because it doesn't matter how accurate the HDR maps may be, they have to be accurately and faithfully reproduced in LDR devices. If the reproduction techniques fail, the whole workflow fails.

In the literature, a number of techniques have been developed for tone reproduction for high contrast images. There are two broad categories of technology, i.e., tone reproduction curve (TRC) based and tone reproduction operator (TRO) based [5]. TRC refers to techniques that manipulate the pixel distributions, representative examples include [8] and 
recent work by the authors $[16,17]$. TRO techniques involve the spatial manipulation of local neighboring pixel values, often at multiple scales, examples include $[10-15]$.

TRO based methods involve multiresolution spatial processing and are therefore computationally expensive. A common difficulty of these techniques is that there are too many parameters the users have to set and this makes them quite difficult to use. In many cases, the setting of these controlling parameters is rather ac hoc and involves many trial and errors. TRC based methods do not involve spatial processing, they are therefore computationally very simple. This is useful in real time applications such as high dynamic range video [4]. These techniques normally have fewer manually adjusted parameters, e.g., methods in [16, 17] have a single adjustable variable. One of the weaknesses of TRC approaches as compared with TRO methods is that it may cause noticeable lost of spatial sharpness in some images. In this paper, we present a new TRC-based tone mapping operator for the visualization of HDR images in LDR devices.

Our development is related to the histogram adjustment technique of [8]. However, the histogram adjustment method of [8] has several drawbacks. Firstly, the algorithm only prevents the display contrast (produced by histogram equalization) from exceeding that of the real scene, and chooses to do nothing about contrast below that of the original scene, therefore nothing will prevent the display from having too low contrast as has also been pointed out in [7]. Moreover, in sparsely populated intensity intervals, compression is achieved by histogram equalization. This means that some sparse areas that span a wide range will be compressed too aggressively. Features in such intervals which are visible to human eyes could be lost. Secondly, if the dynamic range of the scene is already in the device range, the method of [8] uses linear scaling. However, as it is well known that sometimes linear mapping would result in the under utilization of display levels and thus leading to low contrast images, even though the dynamic ranges of the scenes fit within that of the visualization devices. Thirdly, it is too restrictive. In order to produce visually pleasing images to suit different user's subjective preferences, the algorithm should have the capabilities to flexibly adjust the visualization appearance. We hope to overcome these drawbacks and offer a more comprehensive histogram adjustment solution to the mapping of HDR images to LDR images for the purpose of visualization. It should be noted that whilst [8] models human contrast sensitivity, glare, spatial acuity and color sensitivity to exploit the limitations of human vision, we have a simpler goal of developing fast and practical solutions that reproduce optimal visibility for the visualization of high dynamic range radiance maps.

\section{A HIERARCHICAL MAPPING CURVE OPERATOR FOR THE VISUALIZATION OF HDR IMAGES}

One of the keys in mapping HDR image to LDR display is the allocation of displayable levels of LDR devices to HDR pixels. Ultimately, tone mapping entails the assignment of LDR levels to HDR values. If the original scene is well contrast, i.e., its histogram is flat everywhere, then tone mapping can be done by linear scaling. The difficulty comes when the pixels values are unevenly distributed (this is the case in vast majority of natural images and even more so in computer generated graphics). In these images, there are more pixels in one value interval than in others. In other words, across the intensity range, some intervals are densely populated with large amount of pixels falling into the intervals whilst other intervals are sparsely populated with much fewer pixels having these values. The problem with linear scaling is that it treats each interval as equally important regardless of the pixel populations falling into the intervals. On the other hand, the problem of histogram equalization is that it treats the intensity intervals with equal number of pixels falling into them as equally important regardless of the interval's span. A good tone mapping operator should assign more display levels to densely populated HDR intensity intervals whilst give fewer display levels to intensity intervals with smaller pixel populations. Based on this observation, we present a hierarchical nonlinear to linear (HNL) HDR tone-mapping operator.

Instead of mapping the pixels in a single step, HNL is a two-step process. We first divide the HDR intensity range into a predefined number of intervals (in general fewer than the total number of LDR displayable levels). Then each HDR intensity intervals so divided is assigned an appropriate number of display levels. Finally, linear mapping is used to map the HDR intervals onto their corresponding LDR display levels. These two steps, one can be viewed as global and other local and the first step is nonlinear while the second is linear. 
In order to assign an appropriate number of the precious LDR displayable levels, we have developed a simple and effective strategy. The guiding principle for assigning display levels to HDR intervals is that: for densely populated HDR intensity intervals, more display levels should be assigned and for sparsely populated HDR intensity intervals, fewer display levels should be assigned. There are basically two ways to estimate the population density of the intensity interval: either by fixing the intensity intervals' lengths then counting the pixel population falling onto the intervals or by fixing the population size then measuring the intensity spans that cover the same pixel population size. We can then assign display levels according to the pixel density of different intensity intervals, as illustrated in Fig. 1.
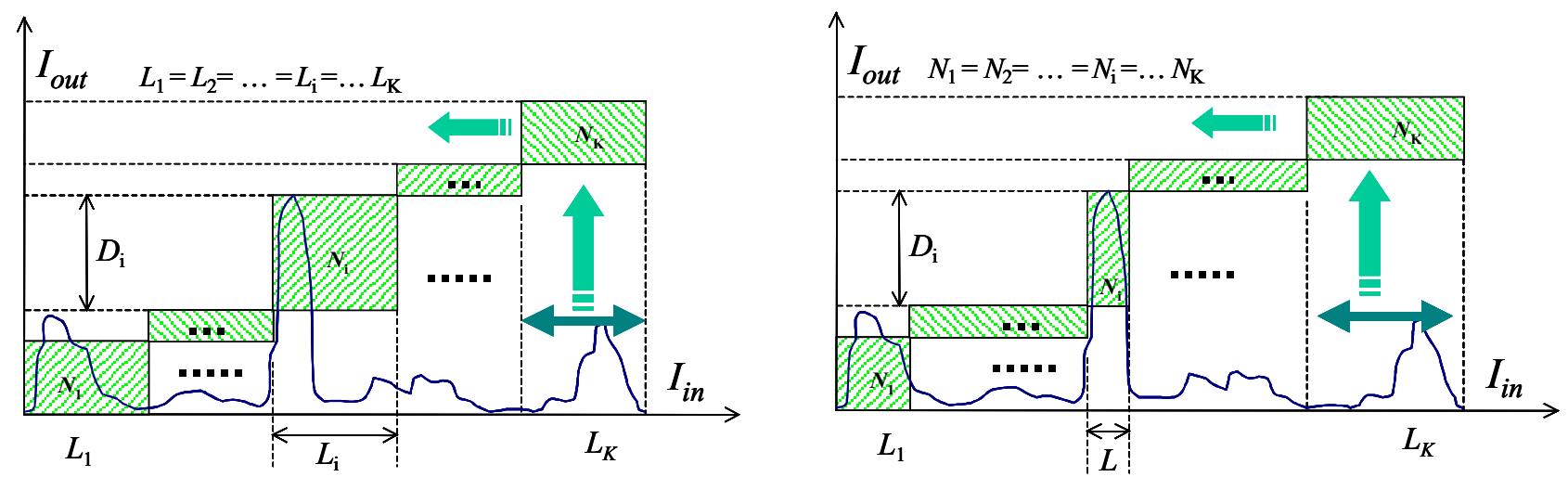

Fig. 1, To estimate the density across the intensity range, we can divide the intensity range into equal length and then count the number of pixels falling into each interval (left). We can also change the length of the interval such that each interval covers the same number of pixels (right). Pixels falling into interval $L_{\mathrm{i}}$ are assigned $D_{\mathrm{i}}$ display levels, which is inversely proportional to $L_{i}$ 's (right) and proportional to $N_{\mathrm{i}}(\mathrm{left})$. In the second step, $L_{\mathrm{i}}$ is linearly mapped to $D_{\mathrm{i}}$

If we divide the HDR intensity into $K$ equal length intervals and the number of pixels falling onto interval $L_{i}$ is $N_{i}$, these $N_{i}$ pixels will then be assigned $D_{i}$ display levels. Obviously, $D_{i}$ should be proportional to $N_{i}$, because the larger $N_{i}$ is, the denser is the interval, and hence more display levels should be assigned to the interval. We found that the following distribution formula worked very well (other similar formulas may also work):

$$
D_{i}=\frac{\left(N_{i}\right)^{\beta_{\lambda}}}{\sum_{\forall k}\left(N_{k}\right)^{\beta_{\lambda}}} D
$$

where $\beta_{\lambda} \geq 0, D$ is the total number of LDR displayable levels. For $\beta_{\lambda}=0$, it is obviously a linear mapping, i.e., equal length intervals of the HDR image's intensity are assigned equal number of display levels in the LDR image. As $\beta_{\lambda}$ increases, relatively more display levels are assigned to denser intervals (larger $N_{i}$ 's) and relatively fewer display levels are assigned to the sparely populated intervals (smaller $N_{i}$ 's). For $\beta_{\lambda}=1$, display levels assigned to the (equal length) HDR intervals are proportional to the pixel populations falling into the intervals. At the extreme, as $\beta_{\lambda} \rightarrow \infty$, all displayable levels will be assigned to the interval with the largest $\tilde{N}_{i}$. Once the number of display levels are assigned to each equal length HDR intensity intervals, pixels in each interval are linearly scaled to fit into the LDR display levels assigned to the interval.

Similarly, we can divide the HDR intensity into $K$ intervals such that each having the same number of pixels falling onto it. Pixels falling into $L_{i}$ will then be assigned $D_{i}$ display levels. In this case, we found that the following distribution formula worked very well (again, other similar formulas may also work):

$$
D_{i}=\frac{\left(L_{i}\right)^{\beta_{\varepsilon}}}{\sum_{\forall k}\left(L_{k}\right)^{\beta_{\varepsilon}}} D
$$

where $\beta_{\varepsilon} \geq 0, D$ is the total number of LDR display levels. For $\beta_{\cdot \varepsilon}=0$, it is obviously histogram equalization mapping, i.e., equal pixel population intervals in the HDR image are assigned equal number of display levels in the LDR image. As $\beta_{\varepsilon}$ increases, relatively fewer display levels are assigned to denser intervals (shorter $L_{i}$ 's) and relatively more display levels are assigned to the sparely populated intervals (longer $L_{i}$ 's). For $\beta_{\varepsilon}=1$, display levels assigned to the (equal 
population) intervals are made proportional to the intervals lengths. At the extreme, as $\beta_{\varepsilon} \rightarrow \infty$, all displayable levels will be assigned to the interval with the longest $L_{i}$. Again, once the number of display levels are assigned to each equal population HDR intensity interval, pixels in each interval are linearly scaled to fit into the LDR display levels assigned to the interval. Fig. 2 plots the relation of $D_{i}$ 's and $L_{i}$ 's or $D_{i}$ 's and $N_{i}$ 's for different values of $\beta_{\varepsilon}$ or $\beta_{\lambda}$. Note (1) and (2) have exact the same expression, but different meanings. Because they have similar performances, our results in this paper use (2).

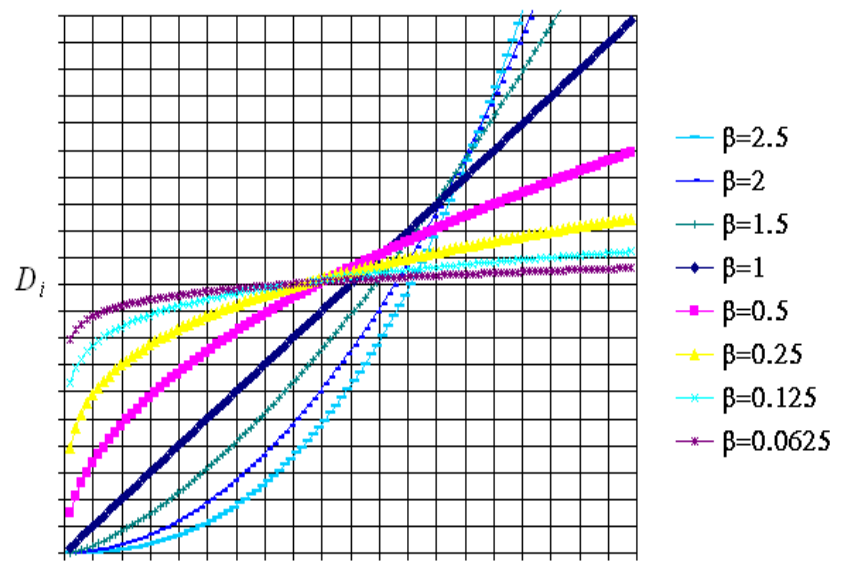

$N_{i}$ or $L_{i}$

Fig. 2, The relation between equal length intervals' pixel population $N_{i}$ 's and the number of display levels assigned to them $D_{i}$ 's for different $\beta_{\lambda}$. The equal population intervals' lengths $L_{i}$ 's and the numbers of display levels $D_{i}$ 's assigned to them for different $\beta_{\varepsilon}$ have the same relation.

Clearly, the HNL operators are computationally highly efficient. The parameter $\beta_{\varepsilon}$ or $\beta_{\lambda}$ has to be determined empirically. However, they both have clear meanings. Since the method is computationally very fast, these parameters can be determined interactively. Our experiences showed that by fixing the number of intervals (1/8 or $1 / 16$ of the total LDR displayable levels worked well), then the operator will have only one variable and finding the appropriate $\beta \mathrm{s}$ is not difficult.

If we want the mapped images to have increased contrast, we can increase $\beta_{\lambda}$ (decrease $\beta_{\varepsilon}$ ). On the other hand, if we want to have reduced contrast, then we can decrease $\beta_{\lambda}$ (increase $\beta_{\varepsilon}$ ). The HNL operator either moves away from linear scaling mapping or moves away from histogram equalization mapping to arrive at an appropriate allocation of the displayable values in LDR devices to the intensity intervals in the HDR images.

\section{HDR IMAGE VISUALIZATION RESULTS}

The technique has been tested on a variety of high dynamic range images. By default, our implementation uses 1,000,000 histogram bins for the HDR images to estimate pixel distribution density and for allocating display levels according to (1) or (2). However, we also found that using histogram bin numbers in the order of $1000 \mathrm{~s}$ our algorithm also work effectively. After mapping, the LDR images are rendered for display and in our implementation, we simply give all pixels mapped to the first interval (lowest values) a display value of 0 , those mapped to the second interval a display value of 1, etc., and those to the last interval (highest values) a intensity value of 255 .

The method is computationally very simple. The parameter can be controlled at an interactive speed even for very large size images thus making the effects of changing the parameters instantly visible. To map an image of $768 \times 512$ pixels on a Pentium 4 with $1800 \mathrm{MHz}$ CPU using non-optimized code, the mapping process takes the HNL operator $0.485 \mathrm{~s}$. In the HNL operator, although there are actually two parameters, the number of intervals in the first step and the exponent $\beta_{\varepsilon}$ or $\beta_{\lambda}$ in (1) or (2). We found that by fixing the number of intervals to 32 or 16 worked well for all images in 
our experiments. After fixing the interval number, then there is only one parameter the user needs to set. Since (1) and (2) are similar, all results presented for the HNL method were implemented based on (2).

\section{APPLICATION TO IMAGE ENHANCEMENT}

With a little imagination, our new HNL operator can be easily applied to enhance ordinary ( 8 bits/pixel grayscale or 24 bits/pixel color) images. Unlike HDR images, these images are distorted by the imaging sensors and in general have low contrast. The purpose is to increase the contrast of these images without introducing objectionable artifacts. Traditionally, this is achieved by histogram equalization. However, traditional histogram equalization stretches the contrast across the entire intensity interval based on the accumulation of pixel populations, which effectively allocating each level with equal number of pixels. In most cases, this processing will introduce unacceptable artifacts. Using our new HNL, we can overcome this difficulty.

Similar to the idea introduced before, if we can sensibly spread out the pixel population across the intensity range such that dense populated intervals are given more display levels and sparsely populated intervals are allocated fewer display levels, and then enhance the contrast of the individual intervals, the enhanced images will not only preserve the originals' visual appearance but also will have enhanced contrast. Effectively, we reassign the image intensities according to their distributions, image intensities that are in a densely populated interval will be given relatively larger ranges and those in sparsely populated intervals will be given shorter ranges. We have used HNL operator to implement a piecewise histogram equalization scheme for low contrast ordinary image enhancement:

Step 1: Divide the intensity range into $N(N<256, N=4$ or 8 worked well in our experience) intervals, by using the first step of the HNL algorithm for the given $N$.

Step 2: For pixels falling into each interval, use traditional histogram equalization to re-map the pixels within the interval. Each interval is histogram equalized independently.

We have experimented our algorithm on a wide range of images for which traditional histogram equalization fails to produce good results. In our experiments, we found that setting $N$ to 4 or 8 worked well. An example is shown in Fig. 5.

\section{CONCLUSIONS}

In this paper, we have presented a computationally efficient and very simple and easy to use high dynamic range image tone mapping technique. Our method is very simple to use. A single parameter, which has a straightforward relationship with the mapped images, controls the appearances of the mapped images in a simple and elegant manner. Experimental results have further demonstrated that the new methods can produce good results on a variety of high dynamic range images. Moreover, our operator can also be used for image enhancement. 

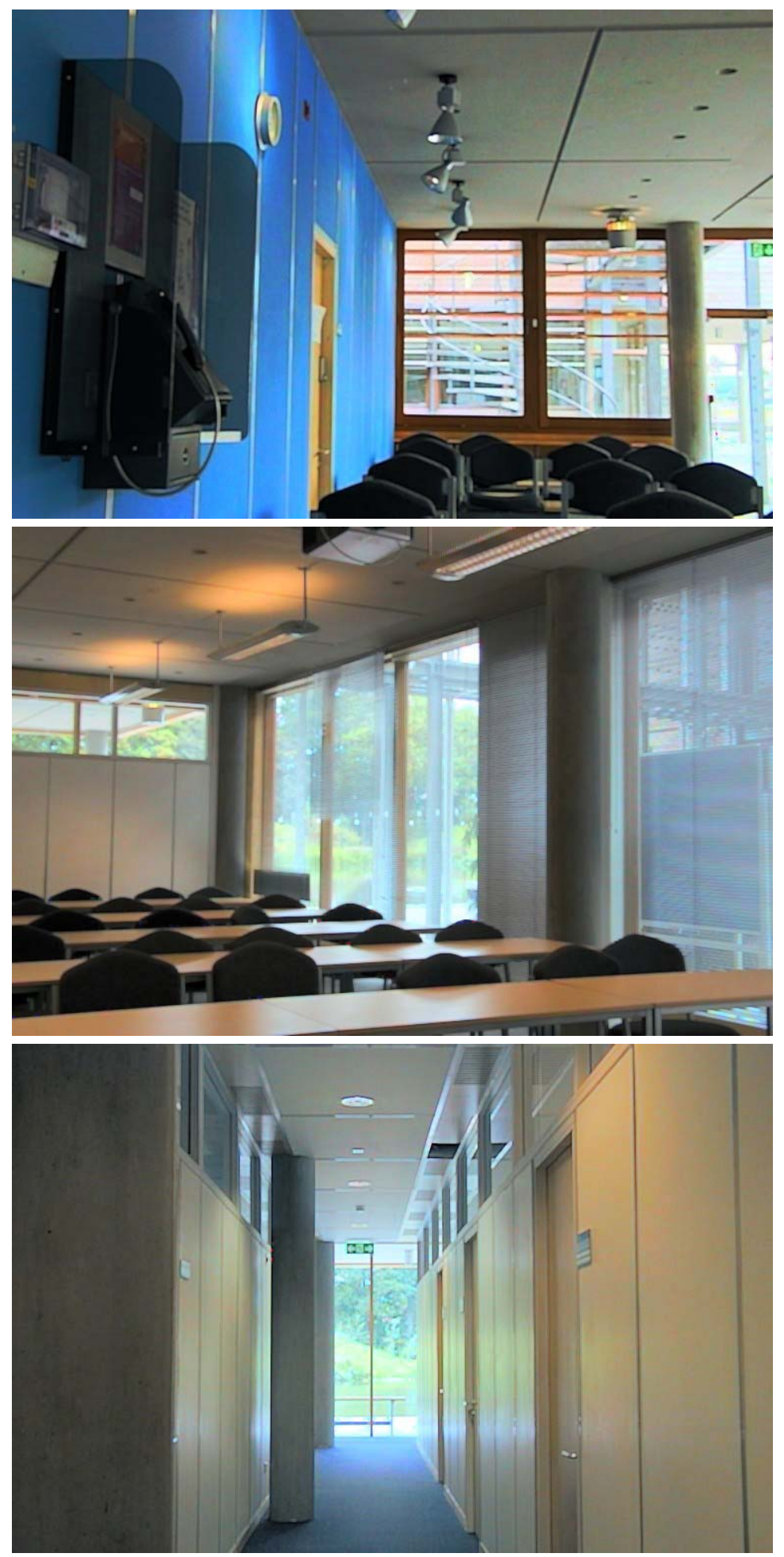

Fig. 3, Results of the HNL high dynamic range image tone mapping operators. Top CS Entrance image, dynamic range of the radiance map is $\mathbf{9 8 , 3 9 5}: \mathbf{1}$. HNL operator parameters: 32 intervals, $\beta_{\varepsilon}=0.5$. Middle: Classroom image, dynamic range of the radiance map is 488,582 : 1. HNL operator parameters, 32 intervals, $\beta_{\varepsilon}=1$. Bottom row: Corridor image, dynamic range of the radiance map is 27,462: 1. HNL operator parameters: 32 intervals, $\beta_{\varepsilon}=1.25$. 

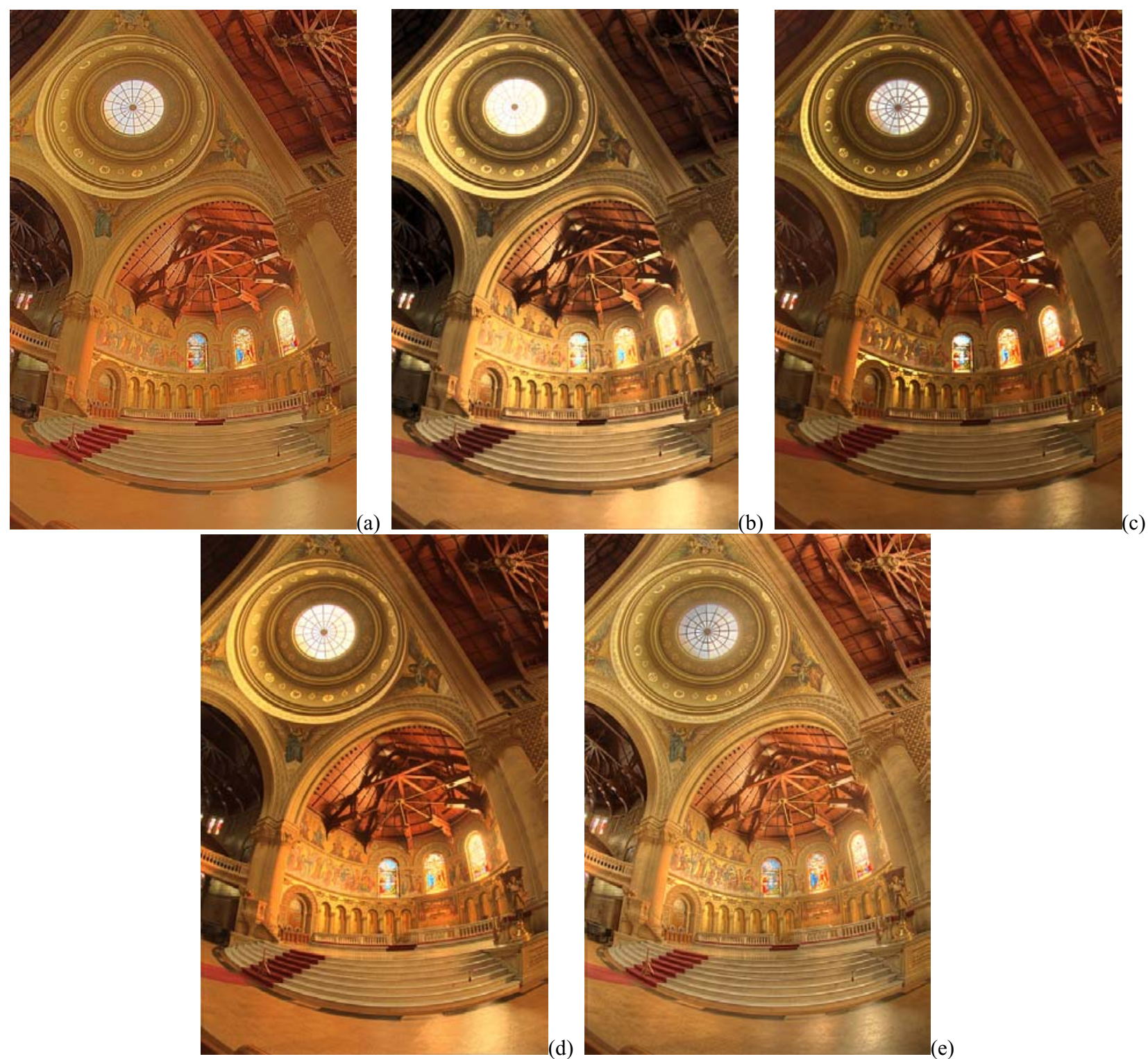

c)

Fig. 4, Tone mapping results of the Memorial Church image. HDR data courtesy of Paul Debevec. (a) Result of our new HNL operator, 32 intervals and $\beta_{\varepsilon}=0.9$. (b) Result of Ward Larson [8]. (c) Result of Bilateral filtering [11]. (d) Result of photographic tone reproduction [12]. (e) Result of the gradient domain method [13]. (b) - (e) are courtesy of Fredo Durand, Dani Lischinski, and Erik Reinhard, printed with permission. 


\section{REFERENCES}

1. P. E. Debevec and J. Malik, "Recovering high dynamic range radiance maps from photographs", Proc. ACM SIGGRAPH'97, pp. $369-378,1997$

2. T. Mitsunaga and S. K. Nayar, "High dynamic range imaging: Spatially varying pixel exposures", Proc. CVPR'2000, vol. 1, pp. 472-479, 2000

3. S. Mann and R. W. Picard, "On being 'undigital' with digital cameras: extending dynamic range by combining differently exposed pictures”, IS\&T's $48^{\text {th }}$ Annual Conference, Society for Imaging Science and Yechnology, Washington D. C., pp. $422-428,1995$

4. S. B. Kang, M. Uyttendale, S. Winder and R. Szeliski, "High dynamic range video", ACM Transactions on Graphics, vol.22, no. 3, Pages: 319 - 325, July 2003

5. J. DiCarlo and B. Wandell, "Rendering high dynamic range images", Proc. SPIE, vol.3965, pp. 392 - 401, 2001

6. J. Tumblin and H. Rushmeier, "Tone reproduction for realistic images", IEEE Computer Graphics and Applications, vol. 13, pp. $42-48,1993$

7. M. Ashikhmin, "A tone mapping algorithm for high contrast images", Proc. Eurographics Workshop on Rendering, P. Debevec and S. Gibson Eds., pp. 1 - 11, 2002

8. G. W. Larson, H. Rushmeier and C. Piatko, "A visibility matching tone reproduction operator for high dynamic range scenes”, IEEE Trans on Visualization and Computer Graphics, vol. 3, pp. 291 - 306, 1997

9. K. Chiu, M. Herf, P. Shirley, S. Swamy, C. Wang and K. Zimmerman, "Spatially nonuniform scaling functions for high contrast images", Proc. graphics Interface'93, pp. 245 - 253, 1993

10. J. Tumblin and G. Turk, "LCIS: A boundary hierarchy for detail preserving contrast reduction", ACM SIGGRAPH 1999

11. F. Durand and J. Dorsey, "Fast bilateral filtering for the display of high-dynamic-range images", Proc. ACM SIGGRAPH'2002

12. E. Reinhard, M. Stark, P. Shirley and J. Ferwerda, "Photographic tone reproduction for digital images", Proc. ACM SIGGRAPH'2002

13. R. Fattal, D. Lischinski and M. Werman, "Gradient domain high dynamic range compression", Proc. ACM SIGGRAPH'2002

14. E. H. Land and J. J. McCann, "Lightness and retinex theory", Journal of the Optical society of America, vol. 61, pp. 1-11, 1971

15. D. J. Jobson, Z. Rahman and G. A. Woodell, "A multiscale Retinex for dridging the gap between color images and the human observation of scenes", IEEE Transactions on Image processing, vol. 6, pp. 965-976, 1997

16. J. Duan, G. Qiu and G. D. Finlayson,"Learning to display high dynamic range images", CGIV'2004, IS\&T's Second European Conference on Color in Graphics, Imaging and Vision, Aachen, Germany, April 5-8, 2004

17. J. Duan and G. Qiu, "Fast Tone Mapping for High Dynamic Range Images", ICPR2004, 17th International Conference on Pattern Recognition, Cambridge, United Kingdom, 23 - 26 August 2004

18. R. M. Gray and D. L. Neuhoff, "Quantization”, IEEE Transactions on Information Theory, vol. 44, no. 6, pp. $2325-2383,1998$

19. D. Marr, Vision, W. H. Freeman and Co., 1982

20. R. Hall, Illumination and color in computer generated imagery, Spinger-Verlag, 1989 


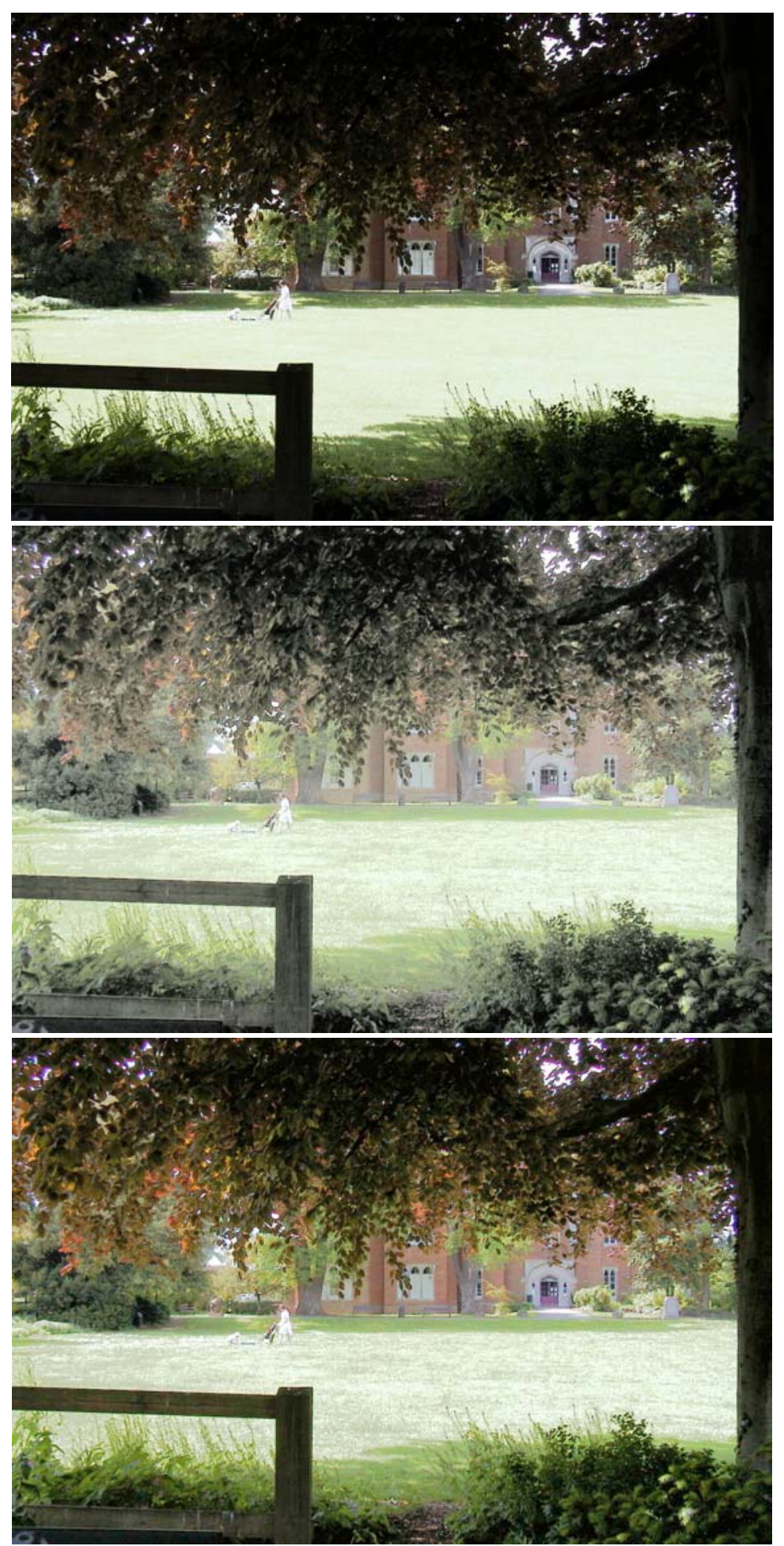

Fig. 5, Image enhancement, Original image (top), traditional (global) histogram equalization (middle) and HNL operator based piecewise histogram equalization. It is seen that global histogram equalization resulted in an image appears "washed-out", the new method overcome such problem while enhancing the image. 